(c) American Dairy Science Association, 2005.

\title{
An Iterative Sensory Procedure to Select Odor-Active Associations in Complex Consortia of Microorganisms: Application to the Construction of a Cheese Model
}

\author{
C. Bonaïti, ${ }^{1}$ F. Irlinger, ${ }^{1}$ H. E. Spinnler, ${ }^{1}$ and E. Engel ${ }^{2}$ \\ 1 UMR INA P-G/INRA Génie et Microbiologie des Procédés Alimentaires, F-78850 Thiverval-Grignon, France \\ ${ }^{2}$ INRA UR Qualité des Produits Aliments, F-63122 Saint-Genès-Champanelle, France
}

\begin{abstract}
The aim of this study was to develop and validate an iterative procedure based on odor assessment to select odor-active associations of microorganisms from a starting association of 82 strains (G1), which were chosen to be representative of Livarot cheese biodiversity. A 3-step dichotomous procedure was applied to reduce the starting association G1. At each step, 3 methods were used to evaluate the odor proximity between mother (n strains) and daughter (n/2 strains) associations: a direct assessment of odor dissimilarity using an original bidimensional scale system and 2 indirect methods based on comparisons of odor profile or hedonic scores. Odor dissimilarity ratings and odor profile gave reliable and sometimes complementary criteria to select G3 and G4 at the first iteration, G31 and G42 at the second iteration, and G312 and G421 at the final iteration. Principal component analysis of odor profile data permitted the interpretation at least in part, of the 2D multidimensional scaling representation of the similarity data. The second part of the study was dedicated to 1 ) validating the choice of the dichotomous procedure made at each iteration, and 2) evaluating together the magnitude of odor differences that may exist between G1 and its subsequent simplified associations. The strategy consisted of assessing odor similarity between the 13 cheese models by comparing the contents of their odor-active compounds. By using a purge-and-trap gas chromatography-olfactory/ mass spectrometry device, 50 potent odorants were identified in models G312, G421, and in a typical Protected Denomination of Origin Livarot cheese. Their contributions to the odor profile of both selected model cheeses are discussed. These compounds were quantified by purge and trap-gas chromatography-mass spectrometry in the 13 products and the normalized data
\end{abstract}

Received May 28, 2004.

Accepted January 8, 2005.

Corresponding author: Erwan Engel; e-mail: erwan.engel@ clermont.inra.fr. matrix was transformed to a between-product distance matrix. This instrumental assessment of odor similarities allowed validation of the choice of G312 as the best 10-strain ecosystem.

(Key words: microbial consortium, cheese model, bidimensional-dissimilarity rating, odor profile)

Abbreviation key: GC-MS = gas chromatographymass spectrometry, GC-O = gas chromatography-olfactometry, $\mathbf{M D S}$ = multidimensional scaling, $\mathbf{P D O}=$ Protected Denomination of Origin.

\section{INTRODUCTION}

The composition of the microbial flora of cheese, its evolution, its activity during ripening, and the interactions between the microorganisms play an important role in the development of the hygienic and sensorial qualities of cheese. The interactions between the flora on the generation of different functions (e.g., flavor, color, taste) have been well documented (Molimard et al., 1994; Bockelman, 2002; Leclercq-Perlat et al., 2004), but were often studied independently, and a rational procedure to generate simultaneously the different functionalities necessary to produce a cheese of high quality has not yet been invented. Most studies focusing on microbial interactions and their sensory consequences aim to explain flavor or texture characteristics of pairs of microorganisms grown as cocultures starting with the properties of a pure culture of the same microorganisms (Molimard et al., 1997; Martin et al., 1999, 2001). In most cases, the generation of a function is dependent on many factors and differs significantly in pure cultures compared with mixed cultures. Indeed, the addition of microorganisms that expressed a specific function in pure culture did not lead necessarily to produce the same function in microbial association. This statement reveals the limitation of an ascending strategy based on the addition of microorganisms. To overcome this limitation, we propose an alternative approach inspired by a previous study dedicated to determining the direct and indirect contribution of taste-active compounds to the 
gustatory properties of various cheeses (Engel et al., 2002a). This 2-step strategy consisted of 1) constructing a cheese model with similar composition and taste properties to the target cheese being studied and 2 ) performing omission tests, which consisted of evaluating the contribution of the components of a mixture to its taste by making a sensory comparison between the taste of the complete cheese model and the taste of a cheese model in which the component was not added. The aim of the present project was to construct a cheese model and to evaluate the contribution of its constitutive strains by omitting some of them. The first step, described in the current paper, was to reduce a cheese model made with 82 selected strains from Livarot cheese to a product made with 10 strains but with similar odor properties.

Specifying differences between samples is a common problem in sensory experiments and the research of one or several discriminative attributes is the classical way to solve it (Matuszewska et al., 1992). In descriptive sensory profiling, each of the samples' attributes is rated for its intensity and a perceptual space is obtained for each sample by representing the scored descriptors as dimensions in space, where the sample is placed in a specific position based on scores given by the panelists (Chauhan and Harper, 1986). However, as well as being time consuming, the use of descriptive techniques raises several problems including the difficulty of choosing from an exhaustive list of independent and relevant terms, and their conceptualization and quantification by a trained sensory panel (Lawless, 1999). To overcome such limitations, several authors have investigated alternative methods. One of the most popular methods consists of measuring product similarity and submitting the data to multidimensional scaling (MDS; Lawless et al., 1995). The output of this procedure is a model reflecting product similarities as proximities of points in space (Moskowitz, 1976). Among other advantages, this approach enables the panelists to rate the overall perceptual dissimilarity of the samples without having to choose or rate descriptive terms (Schiffman et al., 1981) and avoids the problem of individual semantic interpretation of attributes and their definitions (Matuszewska et al., 1992). However, the dimensions of an MDS space are often difficult to interpret (Matuszewska et al., 1992). Comparing profiling and similarity ratings of coffee, Williams and Arnold (1985) found that both procedures resulted in the same grouping of samples. In most cases, the rating scale information may be used together with hedonic measures to interpret the MDS dimensions extracted from similarity rating data.
Table 1. Selected microorganisms used for the making of the cheese model G1. The 82 strains were chosen to be representative of Livarot cheese biodiversity, and the number of strains included in model G1 is shown in parentheses.

\begin{tabular}{ll}
\hline Yeast (33) & Bacteria (49) \\
\hline Geotrichum candidum (20) & Coryneform bacteria (29) \\
Debaryomyces hansenii (4) & Gram-negative bacteria (13) \\
Yarrowia lipolytica (3) & Staphylococci (7) \\
Kluyveromyces lactis (3) & \\
Candida spp. (3) & \\
\hline
\end{tabular}

The first aim of the present study was to reduce, by an iterative procedure based on odor assessment, an association composed of 82 selected strains (G1) into associations of 10 strains. The second objective was 1) to validate the choices made at each iteration of the dichotomous procedure, and 2) to evaluate the magnitude of odor differences that may exist between G1 and its simplified associations using an instrumental evaluation of odor dissimilarity based on the quantification of odor-active compounds.

\section{MATERIALS AND METHODS}

\section{Cheese Models}

Cheese models were made by crushing Livarot curds, manufactured in aseptic conditions by starters, with $22 \%$ of salted sterile water $(1.7 \%$ of $\mathrm{NaCl}$ in the final product). Yeast and bacteria selected were inoculated at $10^{4}$ and $10^{6} \mathrm{cfu} / \mathrm{g}$ of cheese models respectively. Three hundred grams of this medium was placed in a sterile glass box (with a lid), and ripened at $14^{\circ} \mathrm{C}$ for $21 \mathrm{~d}$ in a ripening chamber. The starting association (G1) consisted of 82 strains (33 yeast and 49 bacteria) that were isolated from Livarot cheeses. Table 1 shows the pools of microorganisms used. These strains were chosen from 900 isolates according Bonaïti (2004). Groups G2, G3, G4, and G5 were made of different subgroups of 40 to 43 G1 strains. Similarly, groups G31, G32, G41, and G42 (Figure 1) were made of subgroups of $21( \pm 1)$ G3 or G4 strains, respectively. Groups G311, G312, G421, and G422 were made of subgroups of 10 to 11 strains from G31 or G42. At each step of the dichotomous approach, the subgroups were chosen based on 2 criteria: 1 ) the proportion of the different microbial groups observed in the "mother" cheese model was kept in the reduced consortia, and 2 ) the 2 simplified cheese models with the odor closest to that of the initial cheese model were simplified for a new iteration. At each partition, 5 or 6 different model cheeses were analyzed and compared (Figure 1) after $21 \mathrm{~d}$ of ripening. 


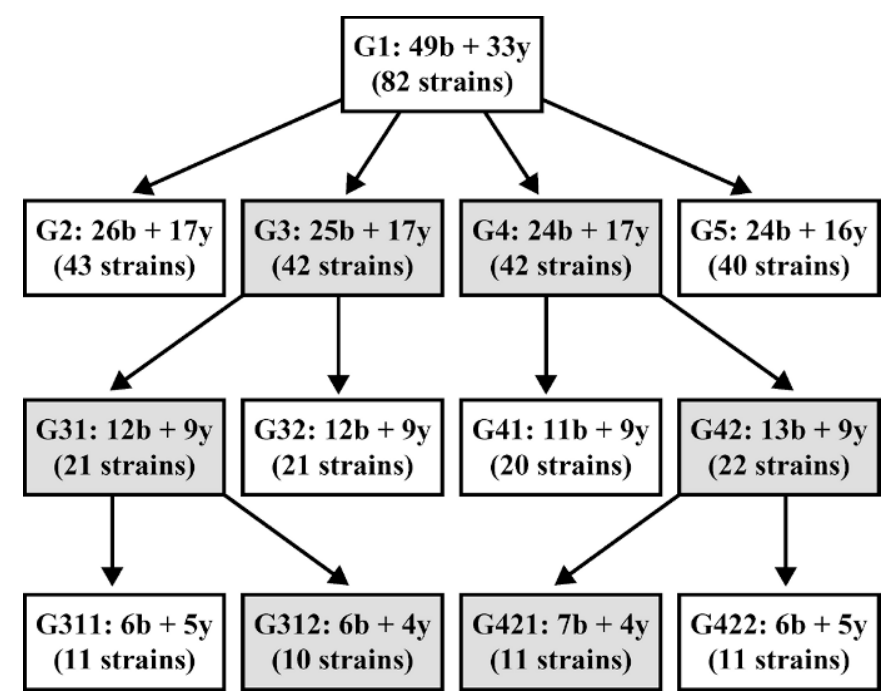

Figure 1. Dichotomous procedure of simplification of model cheeses made with sub-ecosystems of Livarot cheese ( $\mathrm{y}=$ yeast and $\mathrm{b}=$ bacteria).

\section{Odor Assessment}

General conditions. Evaluations were conducted under red light in an air-conditioned room $\left(21 \pm 1^{\circ} \mathrm{C}\right)$. The room was fitted with 10 separated booths according to ISO 45486 . Three 60 -min sessions were organized within a 3 -wk interval. At each session, participants recruited from students and staff of the Thiverval-Grignon center performed successively 3 separate analyses: (1) odor descriptive quantitative profile (indirect measurement); (2) direct dissimilarity assessment; (3) hedonic measurements. Ten volunteers (5 females, 5 males), ranging from 25 to $45 \mathrm{yr}$ of age $($ mean $=35 \mathrm{yr})$ participated in the 3 sessions. The panelists were experienced in odor quantitative descriptive analysis of dairy products and were familiar with the descriptive terms used in this study. However, subjects were naïve with respect to the nature of the stimuli and the objectives of the experiment and had no experience in assessing direct dissimilarity measures.

Regardless of the trial, samples were prepared in the same way. Ten grams of cheese model was placed in an unlabeled glass flask closed with an airtight cap. To ensure the anonymity of the samples, each flask was covered with aluminum foil and coded with a 3digit number. Samples were stored at $4^{\circ} \mathrm{C}$ for $2 \mathrm{~h}$, and then were equilibrated at $21 \pm 1^{\circ} \mathrm{C}$ for $1 \mathrm{~h}$ before odor assessment. For each analysis, panelists were instructed to pause for $20 \mathrm{~s}$ between smelling each sample. Data were recorded using a FIZZ computerized system version 1.20 (Biosystemes, Couternon, France).
Odor quantitative descriptive profile. Two replications of 5 or 6 samples each were assessed at each session. Within each duplicate, samples were presented in a monadic way according to Williams Latin Square design (MacFie et al., 1989) to balance report and position effects. Participants were instructed to smell the products and to evaluate the intensity of descriptors including 9 reference terms of Protected Designation of Origin (PDO) Livarot cheese: ammonia, cowshed, milky, fruity, hazelnut, smoked, pungent, putrid, and sulfur (Institut National des Appellations d'Origine, 1986). If necessary, panelists were able to use additional terms. The panelists assessed the intensity of each descriptor using a 10-cm unstructured linear scale anchored from "no perception" to "strong".

Direct dissimilarity assessment. At each session, the dissimilarity of 5 or 6 cheese model samples was evaluated by the panelists. They were instructed to estimate the overall odor dissimilarity between samples by positioning them in a square paper map of 60 $\mathrm{cm}^{2}$. Samples were presented at the center of the paper. Panelists were permitted to smell samples as many times as they wanted without respecting any specific order. Coordinates were calculated for each sample and each assessor. The largest between-sample distance was used to normalize distances that were obtained from each panelist. Normalized distances were then compiled to obtain an average distance matrix.

Hedonic assessment. At each session, a single series of 5 or 6 model cheese samples was presented to the panelist in a monadic way according to a Williams Latin Square design (MacFie et al., 1989). Panelists were instructed to smell each sample and indicate its hedonic score using a 9-point hedonic scale $(1=$ extremely unpleasant; $5=$ neither like nor dislike; $9=$ extremely pleasant). It is noteworthy that hedonic assessments are generally performed to assess preferences of the global population for a set of products. Such a measurement requires a minimum of 50 assessors. The purpose of the present study was different and hedonic scores were measured only to interpret the product positioning of the 10 panelists according to dissimilarity rating.

\section{Volatile Compounds Analysis by Dynamic Headspace Gas Chromatography- Mass Spectrometry}

Ten grams of cheese model were taken and stored at $-80^{\circ} \mathrm{C}$ until gas chromatography analysis was performed. Before the analysis, samples were thawed at $4^{\circ} \mathrm{C}$, homogenized vigorously, and diluted in water (wt/ 
wt $=1 / 300)$. Sodium chloride $(10 \% \mathrm{wt} / \mathrm{wt})$ was added to the diluted samples. Five milliliters of the mixture was analyzed using a headspace analyzer (HP 7695A purge and trap concentrator; Hewlett-Packard, Palo Alto, CA) coupled to a gas chromatograph (model 6890; Hewlett-Packard, Avondale, PA) equipped with a mass spectrometer detector (HP 5971 quadrupole mass spectrometer; Hewlett-Packard) as previously described by Berger et al. (1999). After homogenization of the sample ( $1 \mathrm{~min}, 1000 \mathrm{rpm}$ ), the 5 -mL sample was transferred to an analytical glass tube for immediate analysis. Each sample tube was connected to the apparatus. After a 2-min prepurge, the tube was heated at $60^{\circ} \mathrm{C}$ for $10 \mathrm{~min}$ and then purged with high purity helium at a flow rate of $30 \mathrm{~mL} / \mathrm{min}$ for $15 \mathrm{~min}$. The volatiles were extracted by adsorption to a porous polymer-adsorbent Tenax trap column (60/80 mesh; 0.25 g; $30 \times 0.32 \mathrm{~cm}$; Tekmar Inc., Cincinnati, $\mathrm{OH})$ at $40^{\circ} \mathrm{C}$. The column was heated at $220^{\circ} \mathrm{C}$ for 2 min to desorb the volatiles, which were transferred at $150^{\circ} \mathrm{C}$ to the head of a capillary column with cryofocusing at $-150^{\circ} \mathrm{C}$. The condensed volatile compounds were analyzed by gas chromatography-mass spectrometry (GC-MS) by heating the interface to $180^{\circ} \mathrm{C}$ for $1 \mathrm{~min}$, followed by automatic splitless injection onto a nonpolar capillary column (HP-5MS; $30.0 \mathrm{~m} \times 0.25 \mathrm{~mm}$; 0.25 $\mu \mathrm{m}$ film thickness) at a helium velocity of $30 \mathrm{~cm} / \mathrm{s}$. The oven temperature was held at $5^{\circ} \mathrm{C}$ for $8 \mathrm{~min}$ and then programmed to rise from 5 to $20^{\circ} \mathrm{C}$ at $3^{\circ} \mathrm{C} / \mathrm{min}$ followed by a rate of $10^{\circ} \mathrm{C} / \mathrm{min}$ to $150^{\circ} \mathrm{C}$ where the temperature was maintained for $10 \mathrm{~min}$. The GC column was connected to a mass-sensitive detector (model 5971 quadrupole mass spectrometer; Hewlett-Packard). The GCMS interface was heated at $280^{\circ} \mathrm{C}$ with the actual temperature in the MS source reaching $180^{\circ} \mathrm{C}$. The electron impact energy was set at $70 \mathrm{eV}$ and data were collected in the range of 29 to 300 atomic mass units at a scan rate of $1.68 \mathrm{scan} / \mathrm{s}$. The same analytical procedure was performed in duplicate for each of the 18 model cheese samples. A $10-\mathrm{mL}$ blank of pure water was analyzed according to the same procedure before and after each sample. Compound identification was based on 1) comparison of retention indices (Van den Dool and Kratz, 1963) with retention indices published (Kondjoyan and Berdagué, 1996) or obtained for pure compounds in our laboratory, 2) mass spectra (MS spectra databases NBS75K and WILEY 275L), and 3) odor properties.

\section{Dynamic Headspace-GC-MS-OIfactometry}

The gas chromatography-olfactometry (GC-O) system consisted of the same GC model as for dynamic headspace GC-MS coupled to a different purge and trap analyzer (3100 sample concentrator, Tekmar Inc.) and mass spectrometer detector (HP 5973, quadrupole mass spectrometer model G1098A; Hewlett Packard). The injection and separation conditions of the volatile compounds were the same. At the end of the column, the effluent was split between a MS and a Sniffer 9000 sniffing device (heated transfer line temperature = $150^{\circ} \mathrm{C}$, humid air $=80 \mathrm{kPa}$; make-up $=20 \mathrm{kPa}$; Brechbühler SA, Switzerland). To determine the odor-active compounds of cheeses, the nasal impact frequency method (Pollien et al., 1997) was performed. Twelve panelists were selected among volunteers in the laboratory. The sniffing sessions were conducted in an airconditioned room $\left(20 \pm 1^{\circ} \mathrm{C}\right)$. Sessions were divided into 2 parts to avoid fatigue. The sessions were planned in such a way that each subject participated in both parts. Panelists recorded the perception of an odor by pressing a button for as long as the component could be smelled. Each panelist was encouraged to describe the odor of each compound detected. Data processing was conducted as described previously (Engel et al. (2002b).

\section{Data Analysis}

Data were processed using release 6.1 of Statistica software (Statsoft, Maisons-Alfort, France). Two-way ANOVA (product, subject) was performed on odor profile data according to the model: attribute $=$ product + subject + product $\times$ subject, with subject treated as a random effect. When significant differences were found $(P<0.05)$, sample mean intensities were compared using Duncan's multiple comparison test. Normed principal component analysis was performed on odor profile data. Direct dissimilarity assessment data were processed by MDS according to an alternative least squares algorithm (ALSCAL) described by Barcenas et al. (2002). For all MDS mapping presented the stress value, which indicates how the data fitted the selected bidimensional solution, was lower than 0.001 .

\section{RESULTS AND DISCUSSION}

\section{Odor Assessment of the Cheese Model}

The aim of the first part of the study was to obtain a cheese model made with a simplified sub-ecosystem of 10 strains. Starting with a complex association of 82 strains (G1), a dichotomous strategy was applied to reduce the microorganisms' number by one-half in each of 3 successive iterations. At each step, odor of cheese models was the criterion used to assess the dissimilarity between mother-daughter associations. 
A

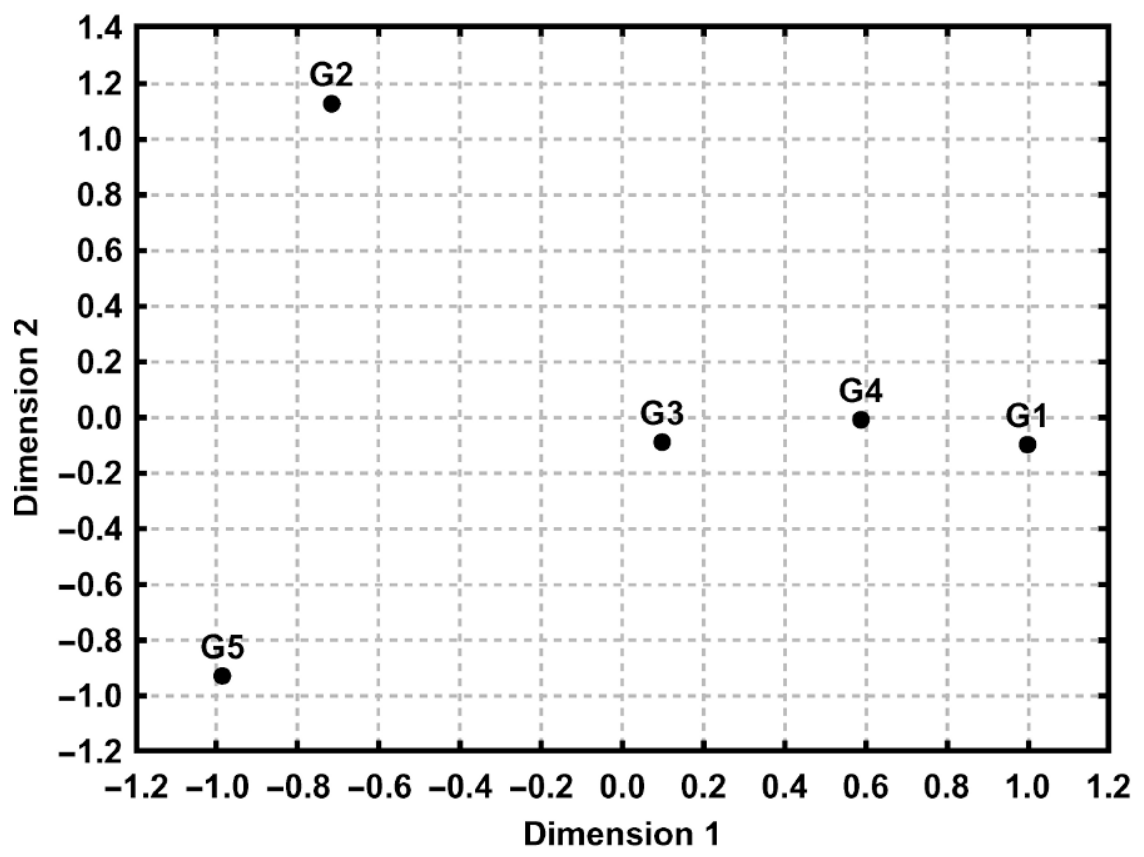

B

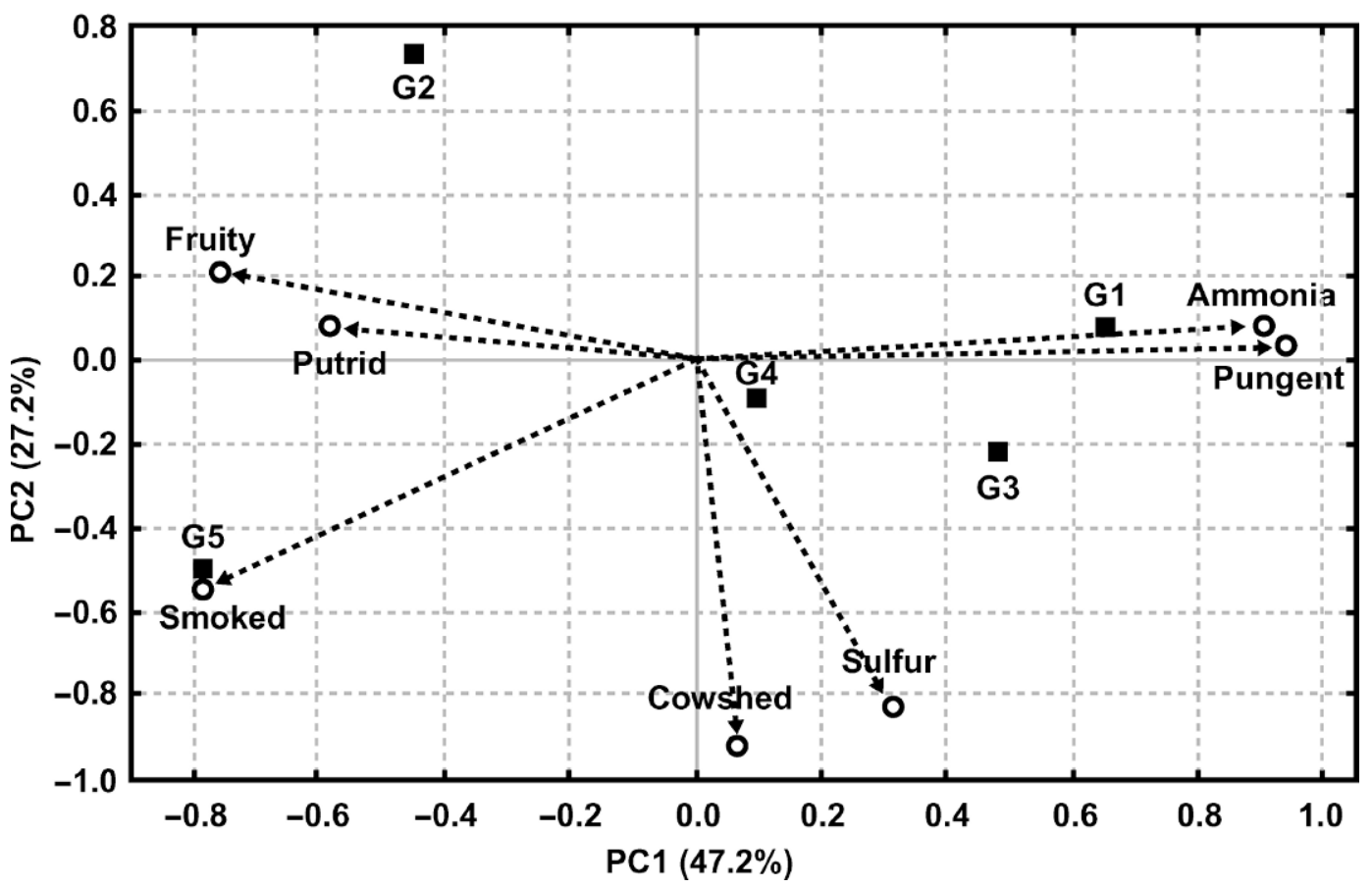

Figure 2. Positioning and characterization of model cheese samples according to their odor at the first step of the dichotomous procedure. (A) 2-D multidimensional scaling solution ( 5 cheese models, stress value < 0.001) based on 2-D odor-dissimilarity assessment; (B) First factorial map of principal components analysis ( 5 cheese models, 7 odor attributes) based on odor profile.

Figure 2A presents the mean arrangement of the products compared by dissimilarity ratings at the first iteration of the dichotomous procedure. According to the weak stress value $(<0.00001)$, the distance matrix was properly summarized on 2 dimensions when the data was processed on an MDS algorithm. The related 
mapping shows that G3 (42 strains) and G4 (41 strains) cheese models are the closest to G1 (82 strains) with respect to their odor. The first principal components explained $74.4 \%$ of variance of the odor profile data of the same products (Figure 2B) confirming the odor similarity between G1, G3, and G4 and their odor difference to G2 and G5. This good agreement between the 2 mapping procedures suggested that the dissimilarity assessment was performed mainly according to qualitative and quantitative criteria. The relative homogeneity of hedonic scores (3.9 to 5.2, not significant according to a 1-way ANOVA, $\alpha=5 \%$ ) agreed with this interpretation. Of the attributes selected (ammonia, cowshed, fruity, pungent, putrid, smoked, sulfur) thanks to a preliminary procedure of data filtering (intensity $>15 / 100$ for at least one product), none differed significantly between the cheese models. This statement suggested that even if the overall odor difference between the products was reliably perceptible, it was difficult to relate it to a particular attribute. The reference associations chosen for the next simplification step were G3 and G4.

The MDS mapping (Figure 3A) of the second set of cheese models (G3, G31, G32, G4, G41, G42) shows a clear discrimination between, on the one hand, G3, G31, G41, and G32, and on the other hand, G4 and G42. Consequently, G42 was preferred to G41. The tight range of hedonic scores (4.2 to 5.5) suggested a limited contribution of this perceptive dimension to the MDS discrimination. In contrast to the G1 to G5 comparison discussed above, the first map of the principal components analysis (Figure 3A) does not show the same between-product arrangement as MDS (Figure 3B), but the first axis reveals an opposition between G3 and G32 according to their fruity (stronger for G3) and putrid (stronger for G32) notes. Two-way ANOVA and Duncan's multiple comparison tests confirmed the significance of these differences $(P<0.005)$. Moreover, discriminative odor attributes did not distinguish between G3 and G31 cheeses. Consequently, G31 and G42 were selected as reference products for the last dichotomous step.

At the final step, the MDS mapping (Figure 4A) points out the proximity between G31 and G312 (versus G311) and between G42 and G421 (versus G422). According to Figure 4B, the first principal components explained $88 \%$ of variance and divided the cheeses into 2 groups: G31, G312, G311, and G42, G421, and G422. According to Table 2, 3 discriminative attributes mainly explain the first principal component: the smoked, sulfur, and fruity notes distinguish the G31, G311, G312 group from the G42, G421, and G422 group. Moreover, significant differences were found in the hedonic scores (Table 2). However, none of the hedonic differences gave any additional argument to better explain the MDS positioning than with the quantitative and qualitative information of odor profile. According to the MDS results, G312 and G421 were the ultimate sub-ecosystem retained by the dichotomous procedure.

The current study demonstrates the usefulness of a dissimilarity rating procedure to discriminate between products according to their overall odor differences and confirms the conclusion of a recent study (Barcenas et al., 2002). The use of this method enables an intuitive positioning of the samples in some psychological space, and contrasts with the complex task of odor profiling which implies 1) the decomposition of the overall space in an exhaustive set of independent and perceptually separable features (Lawless, 1999), 2 ) the choice of proper terms that precisely represent the odor for all panelists (Barcenas et al., 2002), and 3 ) the subjectivity of terms conceptualization. In addition, according to Lawless et al. (1995), similarity data are thought to provide less experimenter influence and to be less constraining to subjects than data from attribute rating scales.

One of the original aims of our study was to perform dissimilarity ratings on a 2-dimensional scale rather than on a classical 1-dimensional scale. This additional dimension enabled the respondents to better reflect the differences between the products. Moreover, results of similarity or sorting data are often summarized on a map constructed with the first 2 MDS dimensions (Matuszewska et al., 1992; Lawless et al., 1995; Barcenas et al., 2002). The bidimensional rating allowed the subject to project the product-space on a map avoiding possible distortion in its statistical reconstruction based on monodimensional rating.

One general limitation of similarity ratings is the necessity of using other sensory procedures to define the dimensions and to describe the main sensory differences among the samples. It is generally admitted that the first dimension to be extracted from similarity data is hedonicity (Moskowitz, 1976). In the present study, this was not relevant. The hedonic assessment performed with only 10 subjects was not intended to characterize the product but only to interpret product positioning according to dissimilarity ratings. The current report suggests that the hedonic differences between the samples are moderate compared with qualitative or quantitative criteria. This hypothesis is consistent with the absence of hedonic discriminative differences between the products (at least in the first 2 dichotomous steps) and, on the other hand, with good agreement between relative product positioning demonstrated with both principal components analysis and MDS at the first and the last step. 
A

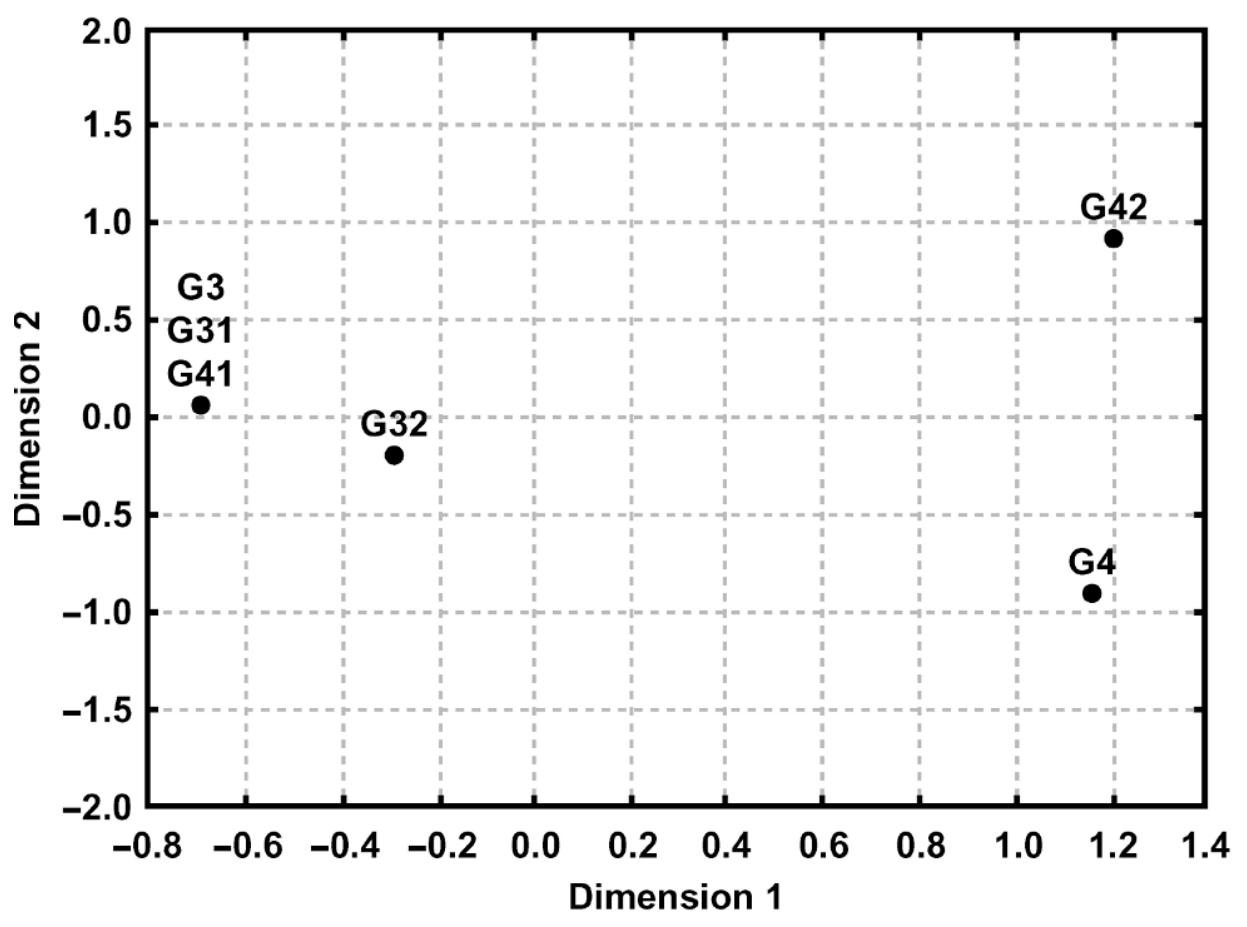

B

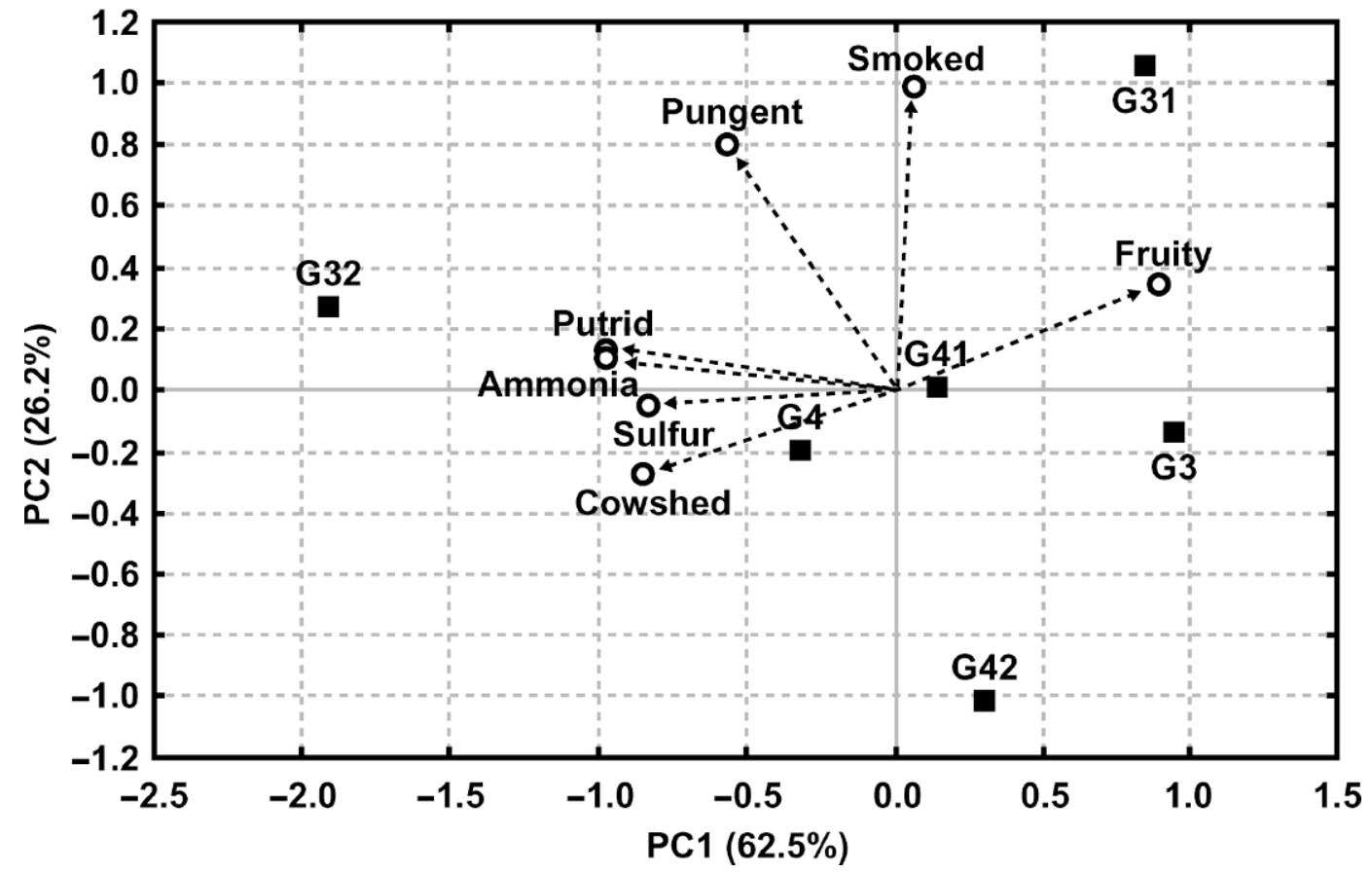

Figure 3. Positioning and characterization of model cheese samples according to their odor at the second step of the dichotomous procedure. (A) 2-D multidimensional scaling solution (6 cheese models, stress value $<0.001)$ based on 2-D odor-dissimilarity assessment; (B) First factorial map of principal components analysis (6 cheese models, 7 odor attributes) based on odor profile. 
Table 2. Mean intensities of the odor profile attributes and hedonic scores of cheese models at the third dichotomous step. ${ }^{1}$

\begin{tabular}{|c|c|c|c|c|c|c|c|c|}
\hline & Smoked** & Fruity** & Sulfur* & Cowshed & Ammonia & Pungent & Putrid & $\begin{array}{l}\text { Hedonic } \\
\text { scores }^{2}\end{array}$ \\
\hline G31 & $\begin{array}{l}20.17 \\
(a b)^{3}\end{array}$ & $\begin{array}{l}39.5 \\
\text { (a) }\end{array}$ & $\begin{array}{l}41.50 \\
\text { (a) }\end{array}$ & 15.75 & 22.25 & 14.50 & 23.33 & $\begin{array}{l}5.71 \\
(\mathrm{bc})\end{array}$ \\
\hline G311 & $\begin{array}{l}37.58 \\
\text { (a) }\end{array}$ & $\begin{array}{l}23.75 \\
(\mathrm{abc})\end{array}$ & $\begin{array}{l}37.17 \\
(\mathrm{ab})\end{array}$ & 18.00 & 19.17 & 13.25 & 9.17 & $\begin{array}{l}5.00 \\
(\mathrm{abc})\end{array}$ \\
\hline G312 & $\begin{array}{l}28.17 \\
\text { (a) }\end{array}$ & $\begin{array}{l}32.0 \\
(\mathrm{ab})\end{array}$ & $\begin{array}{l}31.75 \\
(\mathrm{abc})\end{array}$ & 14.33 & 14.50 & 11.25 & 14.00 & $\begin{array}{l}6.29 \\
\text { (c) }\end{array}$ \\
\hline G42 & $\begin{array}{l}7.83 \\
\text { (b) }\end{array}$ & $\begin{array}{l}16.75 \\
\text { (bc) }\end{array}$ & $\begin{array}{l}26.75 \\
(\mathrm{bc})\end{array}$ & 32.83 & 24.50 & 2.67 & 9.17 & $\begin{array}{l}3.14 \\
\text { (a) }\end{array}$ \\
\hline G421 & $\begin{array}{l}5.50 \\
\text { (b) }\end{array}$ & $\begin{array}{l}9.75 \\
(\mathrm{c})\end{array}$ & $\begin{array}{l}30.08 \\
(\mathrm{abc})\end{array}$ & 31.25 & 29.00 & 6.67 & 18.08 & $\begin{array}{l}4.14 \\
(\mathrm{ab})\end{array}$ \\
\hline G422 & $\begin{array}{l}4.50 \\
\text { (b) }\end{array}$ & $\begin{array}{l}17.5 \\
(\mathrm{bc})\end{array}$ & $\begin{array}{l}19.0 \\
\text { (c) }\end{array}$ & 32.25 & 23.25 & 5.50 & 19.50 & $\begin{array}{l}4.29 \\
(\mathrm{ab})\end{array}$ \\
\hline
\end{tabular}

1Two-way ANOVA (product, subject) was performed on the sensory data set according to the model: attribute $=$ product + subject + product $\times$ subject, on the sensory data $(n=10)$ with subject treated as a random effect. The odor profile was performed twice at each session and by each subject.

${ }^{2}$ Hedonic scores were assessed independently and separately from the odor-profile using a 9-point hedonic scale $(1$ = extremely unpleasant; 5 = neither like nor dislike; 9 = extremely pleasant).

${ }^{3}$ Letters (a,b,c) represent groups resulting from Duncan's multiple comparison tests that were processed on significant attributes and hedonic scores. For each attribute, values are means of individual measurement expressed in percent of the unstructured linear intensity scale anchored from "no perception" to "strong".

$* * P<0.01$ and $* P<0.05$.

Used in a multistep procedure, dissimilarity ratings may have a second disadvantage: the odor proximity between G1 and G3 (respectively, G1 and G4) at the first step, G3 and G31 (respectively, G4 and G42) at the second step, and G31 and G312 (respectively, G42 and G421) at the ultimate step did not necessarily imply an odor proximity between G1 and G312 (respectively, G1 and G421). This lack of transitivity is linked to a property of similarity measurements: their values depend on the products which are compared with and then are just valuable in a well defined product-space. In such an iterative procedure, ratings of sensory attri- butes may be a first solution to follow possible shifts. Starting with the 7 attributes presented in Table 2, a distance matrix was constructed by translating the coordinates of the product in the "attribute ratings" space in between-product Euclidian distances (Table 3). This indirect estimation of odor dissimilarities shows that distance G1 to G312 remained in the same order of magnitude as G1 to G3 and confirmed a limited drift phenomenon. In contrast, these data suggested that the choice of G421 vs. G422 was more ambiguous. The contradiction with the results shown in Figure 4A may be explained in part by the insufficiency of our

Table 3. Assessment of odor dissimilarities between cheese models according to their odor profile attributes ratings. $^{1}$

\begin{tabular}{|c|c|c|c|c|c|c|c|c|c|c|c|c|c|}
\hline & G1 & G2 & G3 & G4 & G5 & G31 & G32 & G41 & G42 & G311 & G312 & G421 & G422 \\
\hline G1 (80) & 0.0 & & & & & & & & & & & & \\
\hline G2 (40) & 20.7 & 0.0 & & & & & & & & & & & \\
\hline G3 (40) & 15.8 & 22.7 & 0.0 & & & & & & & & & & \\
\hline G4 (40) & 15.6 & 20.2 & 5.9 & 0.0 & & & & & & & & & \\
\hline G5 (40) & 24.2 & 21.4 & 17.0 & 15.3 & 0.0 & & & & & & & & \\
\hline G31 (20) & 16.9 & 25.5 & 10.6 & 12.4 & 16.7 & 0.0 & & & & & & & \\
\hline G32 (20) & 27.4 & 23.2 & 30.5 & 30.3 & 32.2 & 29.2 & 0.0 & & & & & & \\
\hline G41 (20) & 14.4 & 17.9 & 9.2 & 9.0 & 19.7 & 12.2 & 25.7 & 0.0 & & & & & \\
\hline G42 (20) & 18.4 & 20.0 & 7.8 & 8.4 & 15.3 & 14.2 & 26.0 & 11.5 & 0.0 & & & & \\
\hline G311 (10) & 12.7 & 14.2 & 11.0 & 9.7 & 15.2 & 11.9 & 22.0 & 7.8 & 9.8 & 0.0 & & & \\
\hline G312 (10) & 13.1 & 15.1 & 9.8 & 9.1 & 15.0 & 11.5 & 22.3 & 7.6 & 8.4 & 1.6 & 0.0 & & \\
\hline G421 (10) & 27.9 & 28.3 & 22.9 & 22.9 & 31.8 & 32.7 & 36.1 & 25.8 & 20.1 & 26.2 & 25.2 & 0.0 & \\
\hline G422 (10) & 13.0 & 16.2 & 7.7 & 6.5 & 14.0 & 10.6 & 25.2 & 7.1 & 7.9 & 3.7 & 3.0 & 24.8 & 0.0 \\
\hline
\end{tabular}

${ }^{1}$ Distance matrix was constructed by translating the coordinates of the product in the "attributes ratings" space in between-product Euclidian distances. The number of strains is shown in parentheses. Depending on iteration, standard deviations of between-products are between 15 and $40 \%$ of Euclidian distance values. Bold values are the Euclidian distances of the associations selected at each iteration. 
A

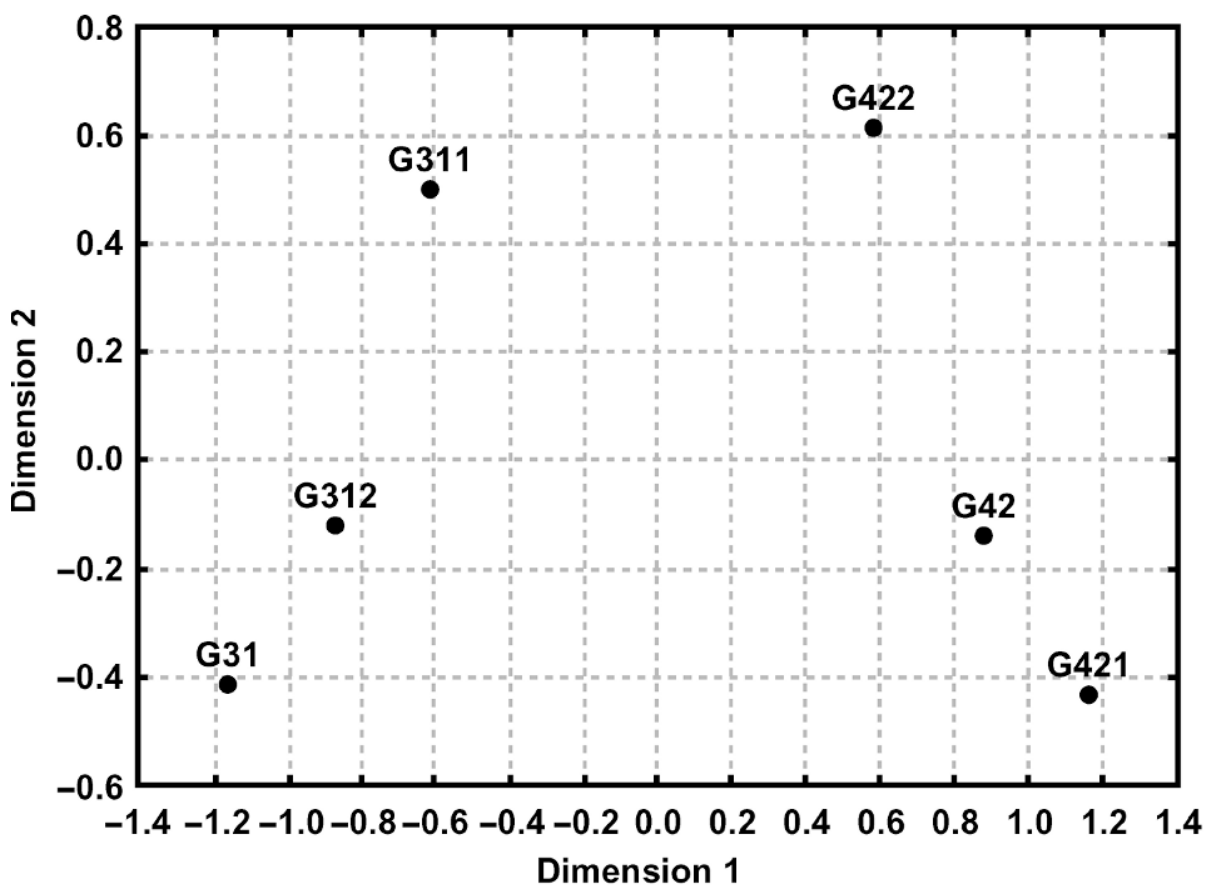

A

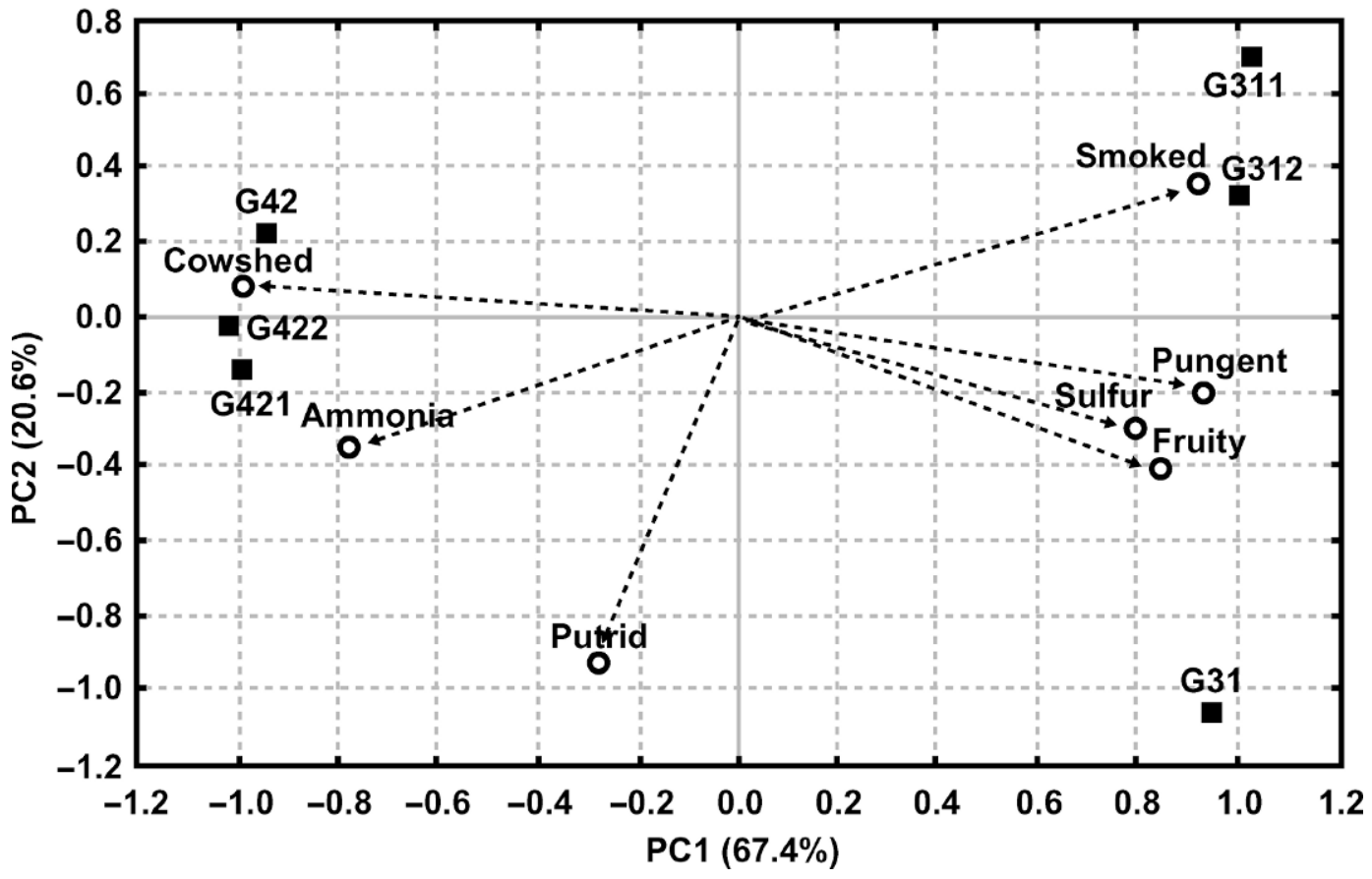

Figure 4. Positioning and characterization of model cheese samples according to their odor at the third step of the dichotomous procedure. (A) 2-D multidimensional scaling solution $(6$ cheese models, stress value $<0.001$ ) based on 2-D odor-dissimilarity assessment; (B) First factorial map of principal components analysis ( 6 cheese models, 7 odor attributes) based on odor profile. 
term list to describe the differences evidenced by the dissimilarity ratings procedure between G422 and the group formed by G42 and G421. Moreover, the wellknown drift in the use of quotation scales by the panelists must complicate a priori the interpretation of profile data compiled from independent odor-profiling sessions (Engel et al., 2001). An alternative strategy to evaluate the between-step drift was then undertaken. It consisted of following (by GC-MS) the evolution of the product-content in compounds responsible for their odor through the whole dichotomous procedure.

\section{Odor-Active Compounds of the Selected Cheese Models (G312 and G421)}

The results of the GC-O analyses performed on G312 and G421 cheese models and on a typical Livarot cheese are summarized in Table 4 . Considering the 3 products together, 50 potentially odor-active compounds were tentatively identified with a MS detector coupled to a GC-O device. Some of the 11 Livarot cheese odorants that were not found in G312 or G421 might explain perceived differences between commercial cheese and cheese models. More particularly, the fruity, nutty, and sulfur notes of some of them may contribute to these distinctive odor features in the typical PDO-Livarot cheese. Among the 28 and 29 compounds identified in G312 and G421, respectively, only 18 were common to both cheese models. This relatively high rate of distinctive odor-active compounds was in agreement with the differences perceived by panelists when they compared directly the odor of both products (Figure 4 and Table 2). Moreover, most compounds allowing the distinction between G312 and G421 were odor-active in Livarot cheese. This last observation suggested that each sub-ecosystem might selectively express complementary parts of the Livarot potent odorants.

One of the main interests in using GC-O/MS was to relate some of the odor attributes of G312 and G421 cheeses to the presence of potentially potent odor-active compounds. Due to their concomitant detection and their sensory proximity, some terms quoted by the sniffers were gathered together in odor families corresponding to attributes used by the panelists to profile the odor of cheese models. Among the discriminative attributes differentiating the cheese models at the last step of the dichotomous procedure (Table 2), the fruity and sulfur notes were quoted by the sniffers to describe several odor-active compounds found in G312 and G421. By taking into account only the compounds with relative quotation frequency values higher than 33\% (Engel et al., 2002b), 3 esters (ethyl butanoate, ethyl hexanoate, and isoamyl acetate) were identified as potential markers for the fruity notes perceived in G312 and G421. Esters have very low odor thresholds: $0.1,1$, and $2 \mathrm{ppb}$ in water for ethyl butanoate, ethyl hexanoate, and isoamyl acetate, respectively (Karahadian and Lindsay, 1985; Molimard and Spinnler, 1996). These compounds are known for their important role in the formation of a fruity character in numerous cheeses (Curioni and Bosset, 2002). Similarly, 5 compounds (methanethiol, methional, dimethyl sulfide, dimethyl disulfide, and dimethyl trisulfide) were identified as potential contributors to the sulfur notes previously quoted by the panelists as one of the main odor features of the cheese models. Their major contribution to the aroma of cheeses could be due to the low value of their perception threshold with respect to their concentration level in these products: $0.02,0.2,0.2,120$, and $0.01 \mathrm{ppb}$ in water for methanethiol, dimethyl sulfide, methional, dimethyl disulfide, and dimethyl trisulfide, respectively (Engel et al., $2002 \mathrm{~b}$ ). Whatever the sulfur compound or the fruity ester, the small relative quotation frequency differences between the 2 products were not sufficient to explain the intensity differences found between the odor profiles of G312 and G421 (Table 2). Starting with the result of numerous odor mixture studies, the odor intensity of esters or sulfur compounds may have been modulated by other compounds with distinct qualitative properties when they are mixed in the product (Laing, 1994; Grosch, 2001). Moreover, the differences of matrix composition and structure may also influence the release of volatile compounds and distort their odor perception (Piraprez et al., 1998).

In contrast with the fruity and sulfur notes, the attributes "smoked" and "putrid" (Table 2) were not used by the sniffers (Table 4). However, the "malty" note that was quoted for 2-methyl-propanal and 3methyl-butanal may match, to some extent, the "smoked" note. The lower relative quotation frequencies in G421 than in G312 was in agreement with the significant differences found when the "smoked" intensity of both products was compared, and strengthens this hypothesis. Similarly, the putrid note might be partly due to the presence of trimethylamine, a compound known for its intense odor of rotten fish as well as for its relatively low perception threshold value estimated at $0.47 \mathrm{ppb}$ of free basic form in water (Molimard and Spinnler, 1996). None of the odor-active compounds listed in Table 4 clearly matches the ammonia and cowshed notes of the cheeses. The ammonia note is generally attributed to the odor of free ammonia derived from amino acid deamination occurring during cheese ripening (Karahadian and Lindsay, 1985). According to the $\mathrm{pH}$ range of the cheese models (7.7 to 8.0), ammonia was mainly present as 
Table 4. Odor activity of volatile compounds identified in Livarot cheese and in the cheese models G312 and G421 made with, respectively, 10 and 11 selected Livarot cheese strains.

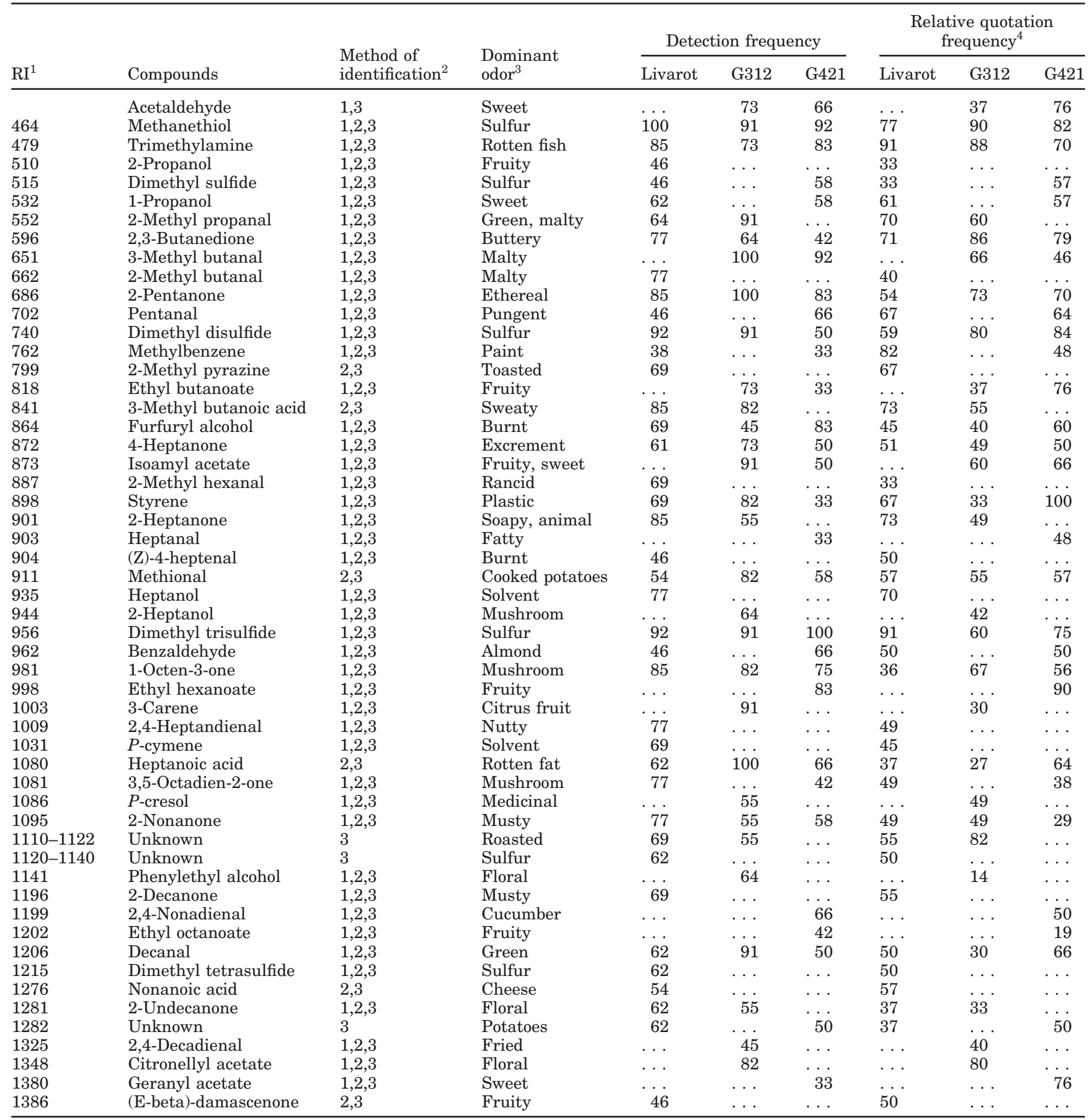

${ }^{1} \mathrm{RI}=$ Retention index on HP-5MS column

${ }^{2} 1$ = Tentative identification using mass spectrometry (MS) and MS databases; $2=$ coincidence between the calculated retention index and known retention index of pure compounds (Kondjoyan and Berdagué, 1996); $3=$ coincidence between odor smelled by the sniffers and known odor of the pure compound.

${ }^{3}$ Most frequently quoted odor family.

${ }^{4}$ Ratio between quotation frequency and detection frequency $(\%)$. 
Table 5. Instrumental assessment of odor dissimilarities between model cheeses (number of strains of each model shown in parentheses). ${ }^{1}$

\begin{tabular}{|c|c|c|c|c|c|c|c|c|c|c|c|c|c|}
\hline & G1 & G2 & G3 & G4 & G5 & G31 & G32 & G41 & G42 & G311 & G312 & G421 & G422 \\
\hline G1 (80) & 0.0 & & & & & & & & & & & & \\
\hline G2 (40) & 11.0 & 0.0 & & & & & & & & & & & \\
\hline G3 (40) & 9.5 & 5.5 & 0.0 & & & & & & & & & & \\
\hline G4 (40) & 7.4 & 9.4 & 8.8 & 0.0 & & & & & & & & & \\
\hline G5 (40) & 13.8 & 12.1 & 13.1 & 6.7 & 0.0 & & & & & & & & \\
\hline G31 (20) & 10.0 & 4.6 & 2.3 & 7.8 & 11.1 & 0.0 & & & & & & & \\
\hline G32 (20) & 4.7 & 9.2 & 5.8 & 7.4 & 13.7 & 6.9 & 0.0 & & & & & & \\
\hline G41 (20) & 8.2 & 7.2 & 2.5 & 9.3 & 14.5 & 4.6 & 4.3 & 0.0 & & & & & \\
\hline G42 (20) & 9.8 & 6.8 & 4.0 & 6.8 & 10.3 & 3.0 & 6.3 & 5.5 & 0.0 & & & & \\
\hline G311 (10) & 5.9 & 6.8 & 4.4 & 7.6 & 13.1 & 5.3 & 3.0 & 3.7 & 5.4 & 0.0 & & & \\
\hline G312 (10) & 12.7 & 7.1 & 6.7 & 7.6 & 8.4 & 4.6 & 9.9 & 8.8 & 4.0 & 8.7 & 0.0 & & \\
\hline G421 (10) & 24.6 & 20.6 & 20.9 & 17.4 & 11.7 & 18.9 & 23.2 & 22.8 & 18.0 & 22.6 & 14.9 & 0.0 & \\
\hline G422 (10) & 9.4 & 5.0 & 2.7 & 8.8 & 12.9 & 3.1 & 5.9 & 3.9 & 3.8 & 4.0 & 6.5 & 21.1 & 0.0 \\
\hline
\end{tabular}

\footnotetext{
${ }^{1}$ Distance matrix was constructed by translating the coordinates of the product in the "odorant content" space in between-product Euclidian distances that "estimates" similarities. Bold values are the Euclidian distances of the "mother" and "daughter" associations selected at each iteration.
}

the free form $\left(\mathrm{pK}=9.30\right.$ at $\left.25^{\circ} \mathrm{C}\right)$. Due to its high volatility and its low molecular weight, this compound was not capable of being detected with our analytical device. Measured by an adapted method (Leclercq-Perlat et al., 2000), the ammonia concentration of similar cheeses ranged from 3 to $10 \mathrm{mg} / \mathrm{g}$ of cheese. With a detection threshold estimated at $90 \mu \mathrm{g} / \mathrm{g}$ of Camembert cheese (Kubickovà and Grosch, 1998a), its contribution to the ammonia note was likely in the cheese model as well as in the Livarot cheese, where its concentration reached $10 \mathrm{mg} / \mathrm{g}$ of cheese. Moreover, this same compound may partly explain the "cowshed" note of the cheese models. None of the attributes found by the sniffers matched with the "cowshed" note of the whole cheese models.

However, p-cresol, which was detected only in G312 and described by about 50\% of the sniffers as "medicinal", has already been associated with "cowy/barny" flavor in Cheddar cheese (Suriyaphan et al., 2001) and may contribute to this flavor note in the model cheeses. However, the absence of p-cresol odor-activity in G421 (Table 4) suggested that the "cowshed" note (which was also perceived as one of the main flavor notes in this model cheese according to Table 2) is probably not only due to this compound. By suggesting that "cowshed" and "ammoniac" may be synonymous, the significant positive correlation between these 2 attributes $(\mathrm{r}=+0.48, \mathrm{n}=72, P<0.05)$ gives a possible explanation.

\section{Instrumental Evaluation of the Odor Similarities Between Cheese Models}

For each step of the dichotomous procedure, the volatile content of every product was analyzed twice with a purge-and-trap GC-MS device. By taking into account only odor-active compounds demonstrated by GC-O, a 1-way ANOVA permitted the selection of the discriminative odorants $(P<0.05)$. After normalization of the data, a distance matrix was constructed by translating the coordinates of the product in the "odorant content" space in between-product Euclidian distances (Table $5)$.

According to their distance to G1, the choice of G3 and G4 was confirmed. Moreover, the compositional differences revealed by the relative positioning of G1, G3, and G4 was in accordance with odor differences observed during odor assessment. At the second step, the tight proximity of G31 (vs. G32) to G3, and to a lesser extent, the close positioning of G42 to G4 (vs. G41) were also coherent with their selection. The distances between G1 and G31 and between G1 and G42 remained in the same range of magnitude as G1 to G3 and G1 to G4. Concerning the ultimate step, the distances G31 to G311 and G31 to G312 being in the same range (respectively, 5.3 and 4.6), the choice of G311 or G312 was equivalent. The fact that the G1 to G312 distance remains in the same range as G1 to G2 or G1 to G5 (Table 5) together with its relative weak value estimated based on attribute ratings (Table 3) suggests a limited drift. In contrast, compositional differences between G42 and its daughter associations together with the comparison between G421 to G1 and G422 to G1 contradicted the choice of G421 and confirm the conclusions based on distances calculated from attribute ratings. The overestimation of the distance G42 to G421 may provide a first explanation. The compounds, which are mainly responsible for compositional differences between G42-G421 and G42G422, are not necessarily the more influential odorants when they are perceived in mixture. However, the distances evaluated directly from attribute ratings 
(Table 3), being in accordance with this dissimilarity between G42 and G421, refute this supposition. Another valid explanation may be the underestimation of the G42 to G422 odor distance due to the possible exclusion of odor-active compounds that would have been detected only in G422 association. The odor-active compounds that were taken into account in the evaluation of "instrumental" distances were selected based on GC-O determination performed on Livarot, G421, and G312, and not on G422. Whatever the arguments, this instrumental estimation of odor-distance perfectly matches the distances assessed based on odor attribute ratings (Table 3), and strengthens the doubt concerning the choice of G42 daughter associations.

Finally, the dichotomous procedure allowed us to select the simplified cheese model G312, which, according to both sensory and instrumental assessment, had an odor similarity comparable to reference association of 82 strains. The next step of our approach will be to perform omission tests on this simplified model mixture to study the contribution of each strain in the mixture to the compositional and sensory perceptions in the cheese.

\section{ACKNOWLEDGMENTS}

This work was financed partly by contract QLK1 CT-2001-02228 from the European Union (Brussels, Belgium). We gratefully acknowledge Marie-Noëlle Leclercq-Perlat for her technical help in the curds manufacturing, Vincent Skowera for technical assistance in GC-O/MS analysis, and all colleagues who participated in the sensory panels. We also thank Tim Cogan for his editorial advice.

\section{REFERENCES}

Barcenas, P., F. J. Pérez Elortondo, J. Salmeron, M. Albisu. 2002. Sensory characterization of ewe's milk cheeses using direct and indirect similarity measures: A comparison. J. Sci. Food Agric. 82:435-442.

Berger, C., N. Martin, S. Collin, L. Gijs, J. A. Khan, G. Piraprez, H. E. Spinnler, and E. N. Vulfson. 1999. Combinatorial approach to flavor analysis. 2. Olfactory investigation of a library of $S$ methyl thioesters and sensory evaluation of selected components. J. Agric. Food Chem. 47:3274-3279.

Bockelmann, W. 2002. Development of defined surface starter cultures for the ripening of smear cheeses. Int. Dairy J. 12:123-131.

Bonaïti, C. 2004. Dynamic approach of functions and microbial interactions in a reconstituted ecosystem by an omission method: Example of the ripening of the Livarot cheese. Thesis of the National Agronomic Institute of Paris-Grignon, France.

Bonaïti, C., and F. Irlinger. 2003. Screening the yeasts and bacteria for aromatic and technological potentialities. Second annual report of SCM project QLK1 CT 2001-02228. 164-176.

Chauhan, J., and R. Harper. 1986. Descriptive profiling versus direct similarity assessments of soft drinks. J. Food Technol. 21:175-187.
Curioni, P. M. G., and J. O. Bosset. 2002. Key odorants in various cheese types as determined by gas chromatography-olfactometry. Int. Dairy J. 12:959-984.

Engel, E., C. Baty, D. Le Corre, I. Souchon, and N. Martin. 2002b. Flavor-active compounds potentially implicated in cooked cauliflower acceptance. J. Agric. Food Chem. 50:6459-6467.

Engel, E., S. Nicklaus, C. Salles, and J. L. Le Quéré. 2002a. Relevance of omission tests to determine flavour-active compounds in food: Application to cheese taste. Food Qual. Pref. 13:505-513.

Engel, E., S. Nicklaus, C. Septier, C. Salles, and J. L. Le Quéré. 2001. Evolution of the taste of a bitter Camembert cheese during ripening: Characterization of a matrix effect. J. Agric. Food Chem. 49:2930-2939.

Grosch, W. 2001. Review. Evaluation of the key odorants of foods by dilution experiments, aroma models and omission. Chem. Senses 26:533-545.

Institut National des Appellations d'Origine (France). 1986. Décret du 29 décembre 1986 relatif à l'appellation d'origine "Livarot". Pages 77-78 in Décrets des produits laitiers AOC. [Recueil de décrets du Journal Officiell. No. CHA00001165.

Karahadian, C., and R. C. Lindsay. 1985. Factors controlling texture in mould surface-ripened cheeses. J. Dairy Sci. 68:92-93.

Kondjoyan, N., and J. L. Berdagué. 1996. Compilation of Relative Retention Indices for the analysis of aromatic compounds. INRA. Laboratoire Flaveur, Saint-Genes-Champanelle, France.

Kubickovà, J. and W. Grosch. 1998a. Evaluation of flavour compounds of Camembert cheese. Int. Dairy J. 8:11-16.

Laing, D. G. 1994. Perception of odor mixtures. Pages 283-297 in Handbook of olfaction and gustation. R. L. Doty, ed. Marcel Dekker, New York, NY.

Lawless, H. T. 1999. Descriptive analysis of complex odors: Reality, model or illusion? Food Qual. Pref. 10:325-332.

Lawless, H. T., N. Sheng, and S. S. C. P. Knoops. 1995. Multidimensional scaling of sorting data applied to cheese perception. Food Qual. Pref. 6:91-98.

Leclercq-Perlat, M.-N., G. Corrieu, H.-E. Spinnler. 2004. The color of Brevibacterium linens depends on the yeast used for cheese deacidification. J. Dairy Sci. 87:1536-1544.

Leclercq-Perlat, M.-N., A. Oumer, F. Buono, J. L. Bergère, H.-E. Spinnler, and G. Corrieu. 2000. Behavior of Brevibacterium linens and Debaryomyces hansenii as ripening flora in controlled production of soft smear cheese from reconstituted milk: Protein degradation. J. Dairy Sci. 83:1674-1683.

MacFie, H. J., N. Bratchell, K. Greenhoff, and L. V. Vallis. 1989 Designs to balance the effect of order of presentation and firstorder carry-over effects in all tests. J. Sens. Stud. 4:129-148.

Martin, N., C. Berger, C. Le Du, and H.-E. Spinnler. 2001. Aroma compounds production in cheese curd by coculturing with selected yeast and bacteria. J. Dairy Sci. 84:2125-2135.

Martin, N., S. Savonitto, P. Molimard, C. Berger, M. Brousse, and H.-E. Spinnler. 1999. Flavor generation in cheese curd by coculturing with selected yeast, mold, and bacteria. J. Dairy Sci. 82:1072-1080.

Matuszewska, I., N. Barylko-Pikielna, L. Tarkkonen, U. Hellemann, and H. Tuorila. 1991-1992. Similarity ratings versus profiling of spreads: Do we need both? Food Qual. Pref. 3:47-50.

Molimard, P., I. Lesschaeve, I. Bouvier, L. Vassal, P. Schlich, S. Issanchou, and H.-E. Spinnler. 1994. Amertume et fractions azotées des fromages à pâte molle de type camembert: Rôle de l'association de Penicillium camemberti avec Geotrichum candidum. Lait 74:361-374.

Molimard, P., I. Lesschaeve, S. Issanchou, M. Brousse, and H.-E. Spinnler. 1997. Effect of the association of surface flora on the sensory properties of mould-ripened cheese. Lait 77:181-187.

Molimard, P., and H.-E. Spinnler. 1996. Review. Compounds involved in the flavor of surface mold-ripened cheeses: Origins and properties. J. Dairy Sci. 79:169-184.

Moskowitz, H. R. 1976. Multidimensional scaling of odorants and mixtures. Lebensm. Wiss. u.-Technol. 9:232-238. 
Piraprez, G., M.-F. Hérent, and S. Collin. 1998. Flavour retention by lipids measured in a fresh cheese matrix. Food Chem. 61:119-125

Pollien, P., A. Ott, F. Montigon, M. Baumgartner, R. Munoz-Box, and A. Chaintreau. 1997. Hyphenated headspace-gas chromatography-sniffing technique: Screening of impact odorants and quantitative aromagram comparisons. J. Agric. Food Chem. 45:2630-2637.

Schiffman, S. S., N. L. Reynolds, and F. V. Young. 1981. Multidimensional scaling: Theory, Methods and Applications. Academic Press, New York, NY.
Suriyaphan, O., M. A. Drake, X. Q. Chen, and K. R. Cadwallader. 2001. Characteristic aroma compounds of British farmhouse Cheddar cheese. J. Agric. Food Chem. 49:1382-1387.

Van den Dool, H., and P. D. Kratz. 1963. A generalization of the retention index system including linear temperature programmed gas liquid partition chromatography. J. Chromatogr. 11:463-471.

Williams, A. A., and G. M. Arnold. 1985. A comparison of the aromas of six coffees characterised by conventional profiling, free-choice profiling and similarity scaling methods. J. Sci. Food Agric. $36: 204-214$ 